\title{
Vertebrates of Tetepare Island, Solomon Islands ${ }^{1}$
}

\author{
Fohn L. Read ${ }^{2}$ and Katherine Moseby ${ }^{3}$
}

\begin{abstract}
Tetepare is the largest unlogged and uninhabited lowland rain-forest island in the South Pacific and is being managed primarily for conservation. An inventory was conducted, and 25 reptile, 4 frog, 76 bird, and 13 mammal species were recorded from Tetepare, including several birds and turtles of international conservation significance. Their relative abundance and local names were collected to assist landowners in attracting researchers and ecotourists and also to develop a conservation management plan for Tetepare.
\end{abstract}

Tetepare Island $\left(8^{\circ} 45^{\prime} \mathrm{S}, 157^{\circ} 32^{\prime} \mathrm{E}\right)$ (Figure 1) is the largest uninhabited island $(11,880$ ha) in the South Pacific. The terrestrial, freshwater, and adjacent marine ecosystems are largely intact due to their isolation from human habitation since the landowners fled Tetepare in the mid-1800s. At the western tip of the island 375 ha were planted with coconuts in 1907-1918, but the rain forest is now reclaiming the plantation, which was progressively abandoned between 1942 and 1990. The common canopy trees of the unlogged predominantly tall lowland forest of Tetepare include Pometia pinnata, Buchanania arborescens, Gmelina moluccana, Vitex cofassus, Celtis latifolia, Intsia bijuga, Calophyllum kajewskii, Heritiera littoralis, and emergent Ficus spp. (Hansell and Wall 1976). Despite the paucity of biological information for the island, Tetepare has been recommended for conservation by J. Diamond (in an unpublished 1976 report on a proposed forest reserve system and conservation strategy for the Solomon Islands), Dahl (1980), SPREP (1985), and Lees (1990).

${ }^{1}$ Worldwide Fund for Nature (Solomon Islands) assisted logistically with this research and provided indemnity from research permits through their Memorandum of Understanding with the Solomon Islands Government. Manuscript accepted 8 March 2005.

${ }^{2}$ Current address: Ecological Horizons, 8 Eyre Court, Roxby Downs, South Australia, 5725.

3 Tetepare Descendants' Association, P.O. Box 131, Munda, Western Province, Solomon Islands.

Pacific Science (2006), vol. 60, no. 1:69-79

(C) 2006 by University of Hawai'i Press

All rights reserved
Customary landowners of Tetepare established the Tetepare Descendants' Association (TDA) in 2002 to oversee resource management and conservation on the island. TDA is promoting sensitive low-level ecotourism as the dominant land use on Tetepare to achieve the joint aims of conservation and income generation for the stakeholders. A newly constructed field station on Tetepare provides a base for researchers, ecotourists, and rangers, by whom an informed resource management plan is being established.

This inventory of the amphibians, reptiles, and birds, with notes on the mammals, of Tetepare Island was conducted by TDA to provide a basis for conservation planning and as an incentive for future biological research on the island. The Tetepare language has almost totally vanished; hence the names of species recorded were sourced from the three closest languages to Tetepare: Roviana, Marovo, and the Touo language of southern Rendova. Recording of these local names will assist guides, researchers, and ecotourists in knowledge sharing and also serve as a valuable written record for each of these languages.

\section{MATERIALS AND METHODS}

Reptiles, amphibians, and birds but not freshwater fish were surveyed opportunistically for a minimum of 2 days and nights in the vicinity of eight campsites on Tetepare from 1999 to 2003 (Table 1). All surveys were conducted in the months from April to December (Table 1). The Lelei area was surveyed in 2002 before construction of the 


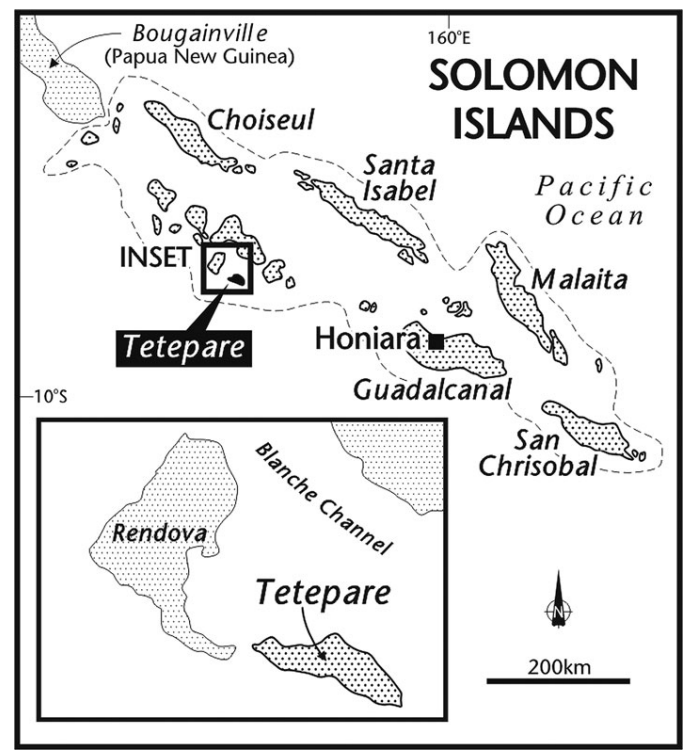

FIgURE 1. Location of Tetepare Island in the Solomon Islands.

Tetepare Field Station and subsequently in 2003 after localized clearing of the regenerating coconut plantation.

All surveys were conducted with the help of local guides. Herptiles (amphibians and reptiles) were searched for by day and night, and records of all small species were verified at least once by capture and identification (McCoy [2000] and Steve Richards from the South Australian Museum). Turtle nests were typically identified by the distinctive tracks of the three local species. Avifauna were recorded by call and sighting. Birds were identified with the aid of binoculars with reference to Beehler et al. (1986) and Doughty et al. (1999), and nomenclature follows Mayr and Diamond (2001). A harp trap and mist nets were used by David Gee to trap bats in the vicinity of Lelei Point in JulyAugust 2003 (Gee 2003). Other bats were captured opportunistically by the authors and identified using Flannery (1995).

This survey did not quantify abundance or densities; however a conservative estimate of individuals observed at each site was recorded to indicate relative abundance and distribution of herptiles and birds. Although recording minimum numbers tends to amplify the numbers of conspicuous broadranging species relative to more cryptic taxa, this technique was considered to be less ambiguous than estimation of abundance classes by observers not initially experienced with the local fauna.

The local name and spelling of all reptiles, amphibians, birds, and mammals recorded on Tetepare were sourced in the field from guides. Additional Touo names were sourced by questioning Henry Gumi, Levan Eroni, and Jonathon Suka in Lokuru. Alfred Bisili from Munda and John Lau from Roviana Lagoon provided additional Roviana names, and Romulus Paoni of Lagoon Lodge and Hviding (1995) were used to confirm Marovo

TABLE 1

Localities and Dates of Fauna Survey Sites on Tetepare Island

\begin{tabular}{lccll}
\hline \hline Camp & Latitude & Longitude & \multicolumn{1}{c}{ Date } & \multicolumn{1}{c}{ Observers $^{a}$} \\
\hline Raro River & $08^{\circ} 42.347^{\prime} \mathrm{S}$ & $157^{\circ} 32.329^{\prime} \mathrm{E}$ & October 1999 & $\mathrm{JR}, \mathrm{KM}, \mathrm{KH}, \mathrm{JT}, \mathrm{EL}$ \\
Fiha Beach & $08^{\circ} 45.661^{\prime} \mathrm{S}$ & $157^{\circ} 33.093^{\prime} \mathrm{E}$ & October 1999 & $\mathrm{JR}$ \\
Keife River & $08^{\circ} 46.730^{\prime} \mathrm{S}$ & $157^{\circ} 39.112^{\prime} \mathrm{E}$ & October 1999 & $\mathrm{JR}$ \\
Qeuru Beach & $08^{\circ} 44.773^{\prime} \mathrm{S}$ & $157^{\circ} 29.709^{\prime} \mathrm{E}$ & April 2001 & $\mathrm{JR}, \mathrm{KM}, \mathrm{MB}, \mathrm{TB}, \mathrm{SU}$ \\
Erava River & $08^{\circ} 42.421^{\prime} \mathrm{S}$ & $157^{\circ} 28.535^{\prime} \mathrm{E}$ & April 2001 & $\mathrm{JR}, \mathrm{KM}, \mathrm{MB}, \mathrm{TB}, \mathrm{SU}$ \\
Tavara & $08^{\circ} 42.192^{\prime} \mathrm{S}$ & $157^{\circ} 26.565^{\prime} \mathrm{E}$ & April 2001 & $\mathrm{JR}, \mathrm{KM}, \mathrm{MB}, \mathrm{TB}, \mathrm{SU}$ \\
Hokata & $08^{\circ} 42.957^{\prime} \mathrm{S}$ & $157^{\circ} 35.097^{\prime} \mathrm{E}$ & August 2002 & $\mathrm{JR}, \mathrm{KM}, \mathrm{MD}, \mathrm{MB}, \mathrm{TB}$ \\
Lelei & $08^{\circ} 43.318^{\prime} \mathrm{S}$ & $157^{\circ} 26.628^{\prime} \mathrm{E}$ & Nov., Dec. 2002 & $\mathrm{JR}, \mathrm{KM}$ \\
Lelei & $08^{\circ} 43.318^{\prime} \mathrm{S}$ & $157^{\circ} 26.628^{\prime} \mathrm{E}$ & June 2003 & $\mathrm{JR}, \mathrm{CF}, \mathrm{CS}, \mathrm{VR}, \mathrm{HL}, \mathrm{WK}, \mathrm{TB}$ \\
& & &
\end{tabular}

a JR, John Read; KM, Katherine Moseby; KH, Keto Hebale; JT, John Tume; EL, Edwin Lau; MB, Mary Bea; TB, Twomey Ben; SU, Suhero; MD, Matthias Daly; CF, Chris Filardi; CS, Catherine Smith; VR, Vanya Rohwer; HL, Hobete; WK, William Kodo. 
names. The local names and suggested spelling of vertebrates occasionally differed between informants. In all cases the names provided by the older informants were subsequently accepted by younger guides or informants.

\section{RESULTS}

Common, scientific, and local names of reptiles, amphibians, birds, and mammals recorded on Tetepare are given in Tables 2-4.

Emoia schmidti was the most abundant of six Emoia species and 25 reptile species re- corded during this survey (Table 5). Other widespread abundant reptiles on Tetepare were the goanna Varanus indicus, the gecko Nactus multicarinatus, and several small Sphenomorphus species (Table 5). Sea snakes (Laticauda spp.), which were not observed but have been reported by guides, would increase the snake inventory to five species. Platymantis solomonis was the most frequently recorded of four frog species (Table 5). Despite limited data from the study sites, both Green (Chelonia mydas) and Hawksbill (Eretmochelys imbricata) Turtles appeared to be relatively abundant in the waters adjacent to Tetepare,

TABLE 2

Touo, Roviana, and Marovo Names of Herptiles from Tetepare

\begin{tabular}{|c|c|c|c|}
\hline Taxon & Touo & Roviana & Marovo \\
\hline \multicolumn{4}{|l|}{ Crocodiles } \\
\hline Crocodylus porosus & Seoto & Basioto & Vua \\
\hline \multicolumn{4}{|l|}{ Goannas } \\
\hline Varanus indicus & Sosi & Regu & Erebachi \\
\hline \multicolumn{4}{|l|}{ Skinks } \\
\hline Corucia zebrata & Bukasi & Bukulu & Bukulu \\
\hline Emoia atrocostata & Fei edo Qore & - & Kaburu kude \\
\hline E. caeruleocauda & Qore & - & Kokojiolo \\
\hline E. cyanura & Qore & - & - \\
\hline E. cyanogaster & Vava kiaka & Kokozialo & Kokojiolo \\
\hline E. nigra & Kudu makau & Gulogulou & Vulouvulou \\
\hline E. schmidti & Qore & Kokozialo & Kokojiolo \\
\hline Lamprolepis smaragdina & Barairi bukasi & Kive & Kokobutongo buma \\
\hline Prasinobaema virens & Barairi bukasi & Kive & - \\
\hline Sphenomorphus bignelli & - & Kokoziolo & Lakuhu \\
\hline S. concinnatus & - & Kilikoso & Kokogilo \\
\hline S. cranei & - & Kilikoso & Kokogilo \\
\hline \multicolumn{4}{|l|}{ Geckos } \\
\hline Nactus multicarinatus & - & Varu razi & - \\
\hline Gehyra oceana & Zaru mata/Tutuombi & Geko & Kumalacha \\
\hline Lepidodactylus guppyi & - & - & Tumajeluku \\
\hline \multicolumn{4}{|l|}{ Snakes } \\
\hline Boiga irregularis & Lelefe & Unara rou & Noki malipara/rou \\
\hline Candoia carinata & Kofutu & Noki putarane/Noki ekekoi & Noki oreke \\
\hline Dendrelaphis salomonis & Visoroqi & Noki tapuru & Noki charava \\
\hline Salomonelaps par & Vasirai ${ }^{1}$ & Noki varipiqei & Noki picha \\
\hline Laticauda sp. & Fei Edonoi & Noki nungununguru pere & Noki nungununguru pere \\
\hline \multicolumn{4}{|l|}{ Turtles } \\
\hline Dermochelys coriacea & Oihare & Tavatolu & Kautolu \\
\hline Eretmochelys imbricata & Safi & Kohale kappa & Vonu pede \\
\hline Chelonia mydas & Foforo & Kohale igana & Vonu ihana \\
\hline \multicolumn{4}{|l|}{ Frogs } \\
\hline Discodeles guppyi & Kurusu & Bakarao & Bangasasa \\
\hline Platymantis solomonis & Kuni & Kuni & Kuni kuni \\
\hline Litoria thesaurensis & Vorandae & Roa & - \\
\hline Ceratobatrachus guentheri & Daka & Daka & Kuchumango \\
\hline
\end{tabular}


TABLE 3

Touo, Roviana, and Marovo Names of Birds from Tetepare

\begin{tabular}{|c|c|c|c|c|}
\hline Common & Scientific & Touo & Roviana & Marovo \\
\hline Little Pied Cormorant & $\begin{array}{l}\text { Phalacrocorax } \\
\text { melanoleucos }\end{array}$ & $\begin{array}{l}\text { Muscuwe } \\
\text { manozo }\end{array}$ & - & - \\
\hline Melanesian Megapode & Megapodius eremita & Ngio & Eo & Io \\
\hline Rufous Night Heron & Nycticorax caledonicus & Qore & Kuarape & Chou chigo \\
\hline Striated Heron & Butorides striatus & Sokodele & Sokodele & Chokodele \\
\hline Black Bittern & Ixobrychus flavicollis & Fiuko & - & Chou chigo \\
\hline Eastern Reef Egret & Egretta sacra & Sou & Soa & Chou \\
\hline Pacific Black Duck & Anas superciliosa & Eqo & Nara & Aranga \\
\hline Crested Hawk & Aviceda subcristata & Tito & Pito & Pito \\
\hline Brahminy Kite & Haliastur indus & Neqa & $\mathrm{Nae}$ & $\mathrm{Ke}$ \\
\hline Solomon Sea-Eagle & Haliaeetus sanfordi & Atao & Atata & Kakaka(pato) \\
\hline Osprey & Pandion baliaetus & Nuru & Manuvu & Chogachoga ingana \\
\hline Variable Goshawk & $\begin{array}{l}\text { Accipiter } \\
\quad \text { novaebollandiae }\end{array}$ & Mano uo & Vari ivu & Tuma/Ivu \\
\hline Pied Goshawk & Accipiter albogularis & - & Kurukuru pella (juv.) & - \\
\hline Purple Swamphen & Porphyrio porphyrio & Bisa & Balikuhu & Bichere \\
\hline Pacific Golden Plover & Pluvialis fulva & Givi & - & Suviviu \\
\hline Mongolian Plover & Charadrius mongolus & - & - & - \\
\hline Gray-tailed Tattler & Tringa brevipes & Hirahira foti & Suviu & Pivivi \\
\hline Whimbrel & Numenius phaeopus & Hirahira foti & Bokala nguzu & Chori minate \\
\hline Beach Thick-knee & Burbinus giganteus & Gio & Bilikiki & Bilikiki \\
\hline Red-necked Stint & Calidris ruficollis & - & Pivivi & - \\
\hline Common Sandpiper & Tringa bypoleucos & Kivi & Pivivi & Chegochego mati \\
\hline Bridled Tern & Sterna anaethetus & - & Bulava & Chelekae \\
\hline Black-naped Tern & Sterna sumatrana & Serekae & Helekae & Chelekae \\
\hline Crested Tern & Sterna bergii & Farao & Vaqolo & Vagolo \\
\hline Black Noddy & Anous minutus & Ofo/Ofongo & Dekere & Dekere \\
\hline Great Frigatebird & Fregata ariel & Amaqi & Belama & Belama \\
\hline Lesser Frigatebird & Fregata ariel & Amaqi & Belama & Belama \\
\hline Brown Booby & Sula leucogaster & Farao & Paraparao & - \\
\hline Nicobar Pigeon & Caloenus nicobarica & Buko & Bakupa & Bakupa \\
\hline Stephan's Ground Dove & Chalcophaps stephani & Buti & Buti & Buti \\
\hline Crested Cuckoo-dove & $\begin{array}{l}\text { Reinwardtoena } \\
\text { crassirostris }\end{array}$ & - & - & Voku \\
\hline $\begin{array}{l}\text { Claret-breasted Fruit- } \\
\text { dove }\end{array}$ & Ptilonopus viridus & Kuvo & Kukuva & Kukuva \\
\hline Superb Fruit-dove & Ptilonopus superbus & Muqi kuvo & Voku & \\
\hline $\begin{array}{l}\text { Red-knobbed Imperial } \\
\text { Pigeon }\end{array}$ & Ducula rubricera & Muqi ngausu & Baruku soloso & Kurukuru/Isu binga \\
\hline Island Imperial Pigeon & Ducula pistrinaria & Kovo kovo & Baruka masa & Kurukuru \\
\hline Cardinal Lory & $\begin{array}{l}\text { Chalcopsitta } \\
\text { cardinalis }\end{array}$ & Siriki & Siri & Chiri \\
\hline Rainbow Lorikeet & $\begin{array}{l}\text { Trichoglossus } \\
\quad \text { baematodus }\end{array}$ & Visuru & Vilisuru & Chiri chaligere \\
\hline Eclectus Parrot & Eclectus roratus & Kara & $\begin{array}{l}\text { Kara siri (fem.), } \\
\text { Kara mahi (m.) }\end{array}$ & $\begin{array}{l}\text { Kara (fem.), } \\
\quad \text { Karamahi (m.) }\end{array}$ \\
\hline Finch's Pygmy-parrot & Micropsitta finschii & - & Kappakappa ngema & Kira kiki \\
\hline Singing Parrot & $\begin{array}{l}\text { Geoffroyus } \\
\text { beteroclitus }\end{array}$ & Kine & Kinkine & Kara kindolo \\
\hline Solomon Cockatoo & Cacatua ducorpsii & Keka & Kakia & Kaka \\
\hline Common Koel & Eudynamys scolopacea & Sengi & Tovao & - \\
\hline Buff-headed Coucal & Centropus milo & $\begin{array}{l}\text { Mozu, Sagaza } \\
\text { (I) }\end{array}$ & Nao, Sengenge (I) & Ao, Chehohu (I) \\
\hline Channel-billed Cuckoo & $\begin{array}{l}\text { Scytbrops } \\
\text { novaehollandiae }\end{array}$ & Barogana & Kukua ranga buko & Paraparao \\
\hline Shining Bronze Cuckoo & Chrysococcyx lucidus & - & - & - \\
\hline
\end{tabular}


TABLE 3 (continued)

\begin{tabular}{|c|c|c|c|c|}
\hline Common & Scientific & Touo & Roviana & Marovo \\
\hline Glossy Swiftlet & Collocalia esculenta & Sidoroi & Pepe rekoho & - \\
\hline Uniform Swiftlet & $\begin{array}{l}\text { Collocalia } \\
\quad \text { vanikorensis }\end{array}$ & Sidoroi & Pepe rekoho & - \\
\hline Common Kingfisher & Alcedo atthis & Siqe & Siqe & Chige \\
\hline $\begin{array}{l}\text { Little/Mangrove } \\
\text { Kingfisher }\end{array}$ & Alcedo pusilla & Siqe & - & - \\
\hline Variable Kingfisher & Ceyx lepidus & Kiu kiu & - & Chige (Pipi) \\
\hline Collared Kingfisher & Halcyon chloris & Siqe & Tarambua & Pipi \\
\hline Beach Kingfisher & Halcyon saurophaga & Kiokio & Kikio & Kiokio \\
\hline Dollarbird & Eurystomus orientalis & Kakahuka & Kikiora & Keke rakocho \\
\hline Blyth's Hornbill & Rbyticeros plicatus & Omehe & Omehe & Omehe \\
\hline $\begin{array}{l}\text { White-throated } \\
\text { Nightjar }\end{array}$ & $\begin{array}{l}\text { Eurostopodus } \\
\text { mystacalis }\end{array}$ & Fehryi & Totoa/Opopotae & Totoa \\
\hline Barn Owl & Tyto alba & Duru duru & Kuarape & - \\
\hline Moustached Tree Swift & Hemiprocne mystacea & Bingehera & Pilisiu & Chiku reta \\
\hline Pacific Swallow & Hirundo tabitica & - & Hikikoro Busa & Keja \\
\hline $\begin{array}{l}\text { White-bellied Cuckoo- } \\
\text { shrike }\end{array}$ & Coracina papuensis & Vizako & Pisale & Pisale \\
\hline $\begin{array}{l}\text { Yellow-eyed Cuckoo- } \\
\text { shrike }\end{array}$ & Coracina lineata & Hote ula & - & Pisale \\
\hline $\begin{array}{l}\text { Melanesian Cuckoo- } \\
\text { shrike }\end{array}$ & Coracina caledonica & Mbisi & - & Matakekeve \\
\hline Common Cicadabird & Coracina tenuirostris & - & - & - \\
\hline $\begin{array}{l}\text { Yellow-vented } \\
\text { Myzomela }\end{array}$ & Myzomela eichborni & $\begin{array}{l}\text { Zeuzeu } \\
\text { Taraqo }\end{array}$ & Buhi siri & Mimili \\
\hline $\begin{array}{l}\text { Common Golden } \\
\text { Whistler }\end{array}$ & $\begin{array}{l}\text { Pachycephala } \\
\text { pectoralis }\end{array}$ & Siosio & Zozovaho & - \\
\hline Willie Wagtail & Rbipidura leucopbrys & Fitikole & Pitikole & Pitikole \\
\hline Rufous Fantail & Rhipidura rufifrons & - & Pepeka erovo & - \\
\hline White-winged Fantail & Rhipidura cockerelli & - & Buhi pitikole & Pitikole \\
\hline White-capped Monarch & Monarcha richardsii & Vingo/Viara & Pakupaku banga & Vivoho \\
\hline $\begin{array}{l}\text { Kolombangara } \\
\text { Monarch }\end{array}$ & Monarcha browni & Viara & Ri zeze & - \\
\hline Steel-blue Flycatcher & Myiagra ferrocyanea & Vie & - & - \\
\hline Tetepare White-eye & $\begin{array}{l}\text { Zosterops rendovae } \\
\text { tetiparius }\end{array}$ & Siokoli & Pika & Chikubusa \\
\hline Yellow-bellied Sunbird & Nectarinia jugularis & Vihu & Hilihiliboe & Viu \\
\hline Yellow-faced Myna & Mino dumontii & Fisikoli & Kinio & Kolioro \\
\hline Singing Starling & Aplonis cantoroides & Neo & Mata kekeve & Chichiu \\
\hline Metallic Starling & Aplonis metallica & Neo & Hiuheze & Ea (Chichiu) \\
\hline Brown-winged Starling & Aplonis grandis & Mbissi & Hindoko & - \\
\hline
\end{tabular}

and Leatherbacks (Dermochelys coriacea) were the most frequently recorded turtle species nesting on Tetepare's Qeuru, Tofa, and Keife beaches.

A total of 72 bird species (Table 6) was recorded from Tetepare Island during this survey. Four other species can be added to this inventory. The Black Bittern (Ixobrychus flavicollis) was recorded by J. Diamond (pers. comm.), and Great Frigatebird (Fregata ariel) and Channel-billed Cuckoo (Scythrops novae- bollandiae) were recorded on Tetepare by G. Dutson (pers. comm.). Sacred Kingfishers (Halcyon sancta) could not be confidently identified in the field but are likely to be migratory visitors to Tetepare during the austral winter and were recorded by C. Filardi and C. Smith (pers. comm.). Thirteen mammal species (Table 4) have been recorded from this incomplete mammal survey of Tetepare.

Each of the languages used different names for adult and juvenile Buff-headed Coucal 
TABLE 4

Touo, Roviana, and Marovo Names of Mammals from Tetepare

\begin{tabular}{llccc}
\hline \hline Common & \multicolumn{1}{c}{ Scientific } & Touo & Roviana & Marovo \\
\hline Large Flying Fox & & Rano suri & Veke taluaba & Samuru \\
"Hissing" Flying Fox & & Erueru suri & $\begin{array}{l}\text { Veke sui } \\
\text { Veke lagiso }\end{array}$ & $\begin{array}{l}\text { Lahu } \\
\text { "Kissing, Flying Fox }\end{array}$ \\
Solomons Bare-backed Fruitbat & Dobsonia inermis & Hovi & - & - \\
Small Melanesian Bent-winged Bat & Miniopterus macrocneme & - & - & - \\
Common Rousette Bat & Rousettus amplexicaudatus & Eru eru & - & - \\
Northern Blossom-bat & Macroglossus minimus & - & - & - \\
Fardoulis' Blossom-bat & Melonycteris fardoulisi & - & - & Tataemoa \\
New Guinea Pipistrelle & Pipistrellus angulatus & Rika & Peperekoho & - \\
Solomon Island Tube-nosed Bat & Nyctimene bougainville & - & - & - \\
Horseshoe-bat & Hipposideros maggietaylorae & - & - & Rumu \\
Dugong & Dugon dugon & Vena & Manue & Binahere \\
Cuscus & Phalanger orientalis & Odo & Mutu \\
Small Rat & Rattus sp. & Siro & Gilgale & Moa \\
Pig & Sus scrofa & Bo & Boko & - \\
Cat & Felis catus & - & - & - \\
\hline
\end{tabular}

(Centropus milo), and all guides questioned considered that the juvenile birds were a separate species from the differently colored adults. In contrast, locals used a single name for several groups of similar taxa, such as several of the small skinks, small waders, and swiftlets. Many of the local names were onomatopoeic, including the frogs Kuni and Roa and the birds Halcyon saurophaga (Kiokio) and Cacatua ducorpsii (Kaka). The local names of some species either had been forgotten or were not known by some observers. Most unnamed species were either rare nomads or migrants (Reinwardtoena crassirostris, Chrysococcyx lucidus, Charadrius mongolus) or cryptic species (Alcedo pusilla, Accipiter albogularis, Coracina tenuirostris). Due to the similarity of the two languages, it is not surprising that the Roviana and Marovo names are often identical or similar. It is apparent that some of the contemporary Touo names, in particular, have been adopted from Roviana names of similar species.

\section{DISCUSSION}

Although species inventories are possibly incomplete for many of the Solomon Islands, the 21 terrestrial reptile species recorded on Tetepare exceeded that recorded for the nearby similar-sized islands of Vangunu (15), Ranonga (14), Rennell (13), and Kolombangara (11) (McCoy 2000). Five more reptile species have been recorded from the large nearby island of New Georgia compared with Tetepare, with the greater richness of geckos $(7: 3)$ and blind snakes (2:0) being the main difference between the two reptile faunas (McCoy 2000). The much larger islands of Malaita (29) and Guadalcanal (39) and especially Bougainville (48) support a richer reptile fauna than any of the islands in the Western Province.

Leatherback Turtles (Dermochelys coriacea), which nest on Tetepare, have been predicted to decline to extinction in the South Pacific within the next decade (Spotila et al. 2000). Scavenging of nests by abundant Mangrove Monitors (Varanus indicus), harvesting by hunters, and inundation by high tides appear to inflict considerable mortalities on Tetepare's turtle nests (pers. obs.). Green Turtles (Chelonia mydas) also nest on Tetepare's beaches, and up to 16 individuals were recorded foraging in the sea-grass beds on the western and southern coasts. These sea-grass beds, particularly in the sheltered lagoon at the western extremity of Tetepare, were also used by an apparently resident Dugong $(D u-$ gon dugon) population. Local divers reported 
TABLE 5

Qualitative Abundance of Herptiles from Eight Fauna Survey Sites on Tetepare

\begin{tabular}{|c|c|c|c|c|c|c|c|c|c|}
\hline & Rano & $\begin{array}{l}\text { South } \\
\text { beach }\end{array}$ & Kiefe & Qeuru & Erava & Tavara & Hokata & $\begin{array}{l}\text { Lelei } \\
2002\end{array}$ & $\begin{array}{l}\text { Lelei } \\
2003\end{array}$ \\
\hline \multicolumn{10}{|l|}{ Crocodiles } \\
\hline Crocodylus porosus & & $1^{a}$ & & 1 & & 1 & & & \\
\hline \multicolumn{10}{|l|}{ Goannas } \\
\hline Varanus indicus & $5+$ & $5+$ & $5+$ & 2 & 2 & 3 & 8 & 2 & 1 \\
\hline \multicolumn{10}{|l|}{ Skinks } \\
\hline Corucia zebrata & & 1 & & & 1 & & & & \\
\hline $\begin{array}{l}\text { Emoia atrocostata } \\
\text { E. caeruleocauda }\end{array}$ & $1^{a}$ & & 5 & 4 & 1 & 1 & & 2 & 2 \\
\hline E. cyanura & 1 & & & & & 1 & & & \\
\hline E. cyanogaster & & 2 & & & & 1 & & 1 & \\
\hline E. nigra & 1 & & 2 & & & 1 & 2 & 1 & 2 \\
\hline $\begin{array}{l}\text { E. schmidti } \\
\text { Lamprolepis smaragdina }\end{array}$ & $20+$ & $\begin{array}{c}20+ \\
1\end{array}$ & $20+$ & 13 & $\begin{array}{l}6 \\
1\end{array}$ & $50+$ & & $\begin{array}{c}20+ \\
2\end{array}$ & $10+$ \\
\hline Lipinia noctua & & & & & & & & & 1 \\
\hline Prasinohaema virens & & 4 & & & & & & 1 & \\
\hline Sphenomorphus bignelli & $10+$ & & & & 1 & & 4 & 1 & 3 \\
\hline S. concinnatus & 5 & 2 & & & 1 & & 2 & 7 & 4 \\
\hline S. cranei & 1 & & & & & 2 & 2 & & \\
\hline \multicolumn{10}{|l|}{ Geckos } \\
\hline Nactus multicarinatus & 7 & 2 & 5 & 2 & 1 & 5 & 1 & & \\
\hline Gebyra oceana & & 2 & & 1 & 2 & & & 1 & 2 \\
\hline Lepidodactylus guppyi & & 1 & 1 & & & & & & \\
\hline \multicolumn{10}{|l|}{ Snakes } \\
\hline Boiga irregularis & & & & & 1 & & & 1 & 1 \\
\hline Candoia carinata & 1 & & & & 1 & & & & 2 \\
\hline Dendrelaphis salomonis & $1^{a}$ & & & & & & & & 1 \\
\hline Salomonelaps par & 2 & 2 & 1 & 2 & & 1 & & & \\
\hline \multicolumn{10}{|l|}{ Turtles } \\
\hline Dermochelys coriacea & & $2^{a}$ & & $1(\mathrm{n})^{b}$ & & & & & \\
\hline Eretmochelys imbricata & & 1 & & & & 1 & & & \\
\hline Chelonia mydas & & & & & & & 1 & & 1 \\
\hline \multicolumn{10}{|l|}{ Frogs } \\
\hline Discodeles guppyi & 1 & $5+$ & $1+$ & 5 & 1 & & 1 & & \\
\hline Platymantis solomonis & $10+$ & $10+$ & $10+$ & $100+$ & 30 & 2 & 10 & 2 & 5 \\
\hline Litoria thesaurensis & 1 & & & & 1 & 3 & & & \\
\hline Ceratobatrachus guentheri & 2 & & & 15 & 5 & 1 & 4 & & 3 \\
\hline
\end{tabular}

${ }^{a}$ Recorded $>1 \mathrm{~km}$ from campsite.

${ }^{b}$ Nesting.

frequent encounters with Dugong including calves in this lagoon. Hawksbill Turtles (Eretmochelys imbricata) were frequently recorded in the reefs around the more sheltered northern coast of Tetepare.

The Prehensile-tailed Skink (Corucia zebrata), endemic to the Solomon Islands, is reportedly the largest skink in the world. The Prehensile-tailed Skink is also unusual in being arboreal, herbivorous, and nocturnal. Estimations of abundance of this cryptic species in undisturbed rain forest are problemat- ical, because most C. zebrata on other islands are found when trees are felled. McCoy (2000) considered that wild populations of $C$. zebrata, along with Varanus indicus and Candoia spp., are vulnerable to extinction from unregulated collection for the international pet trade. Protection of these three taxa on Tetepare may therefore be of national importance. Another interesting arboreal skink found on Tetepare is Prasinobaema virens, one of the few vertebrates in the world with green blood (Austin and Jessing 1994). 
TABLE 6

Minimum Counts of Birds Recorded from Fauna Survey Sites on Tetepare

\begin{tabular}{|c|c|c|c|c|c|c|c|}
\hline Common Name & 1999 & Qeuru & Erava & Tavara & Hokata & Lelei 2002 & Lelei 2003 \\
\hline Little Pied Cormorant & & & & 2 & & & \\
\hline Melanesian Megapode & *(b) & 1 & & 2 & 2 & 2 & 3 \\
\hline Rufous Night Heron & *(b) & 1 & & 2 & & 1 & \\
\hline Striated Heron & * & & 2 & 2 & 1 & 1 & 1 \\
\hline Eastern Reef Egret & * & 4 & & & 1 & 3 & 1 \\
\hline Pacific Black Duck & * & & 1 & 5 & 2 & 9 & 9 \\
\hline Crested Hawk & * & 1 & 2 & 2 & 3 & & \\
\hline Brahminy Kite & * & 1 & 3 & 2 & & 2 & \\
\hline Solomon Sea-Eagle & * & 1 & 2 & 1 & 1 & 2 & 1 \\
\hline Osprey & * & 1 & & 1 & & 1 & 2 \\
\hline Variable Goshawk & & & & 1 & & & 2 \\
\hline Pied Goshawk & & & & & & & $1^{*}$ \\
\hline Purple Swamphen & * & & 1 & 1 & & 1 & 2 \\
\hline Pacific Golden Plover & * & 3 & & & & & \\
\hline Mongolian Plover & * & 3 & & & & & \\
\hline Gray-tailed Tattler & * & 5 & & 6 & & & \\
\hline Whimbrel & * & 1 & & 1 & & 1 & \\
\hline Beach Thick-knee & * & 2 & & 2 & & 2 & 1 \\
\hline Red-necked Stint & * & & & & & & \\
\hline Common Sandpiper & * & & 1 & & 3 & 1 & \\
\hline Bridled Tern & * & & & & & & \\
\hline Black-naped Tern & * & & & & 5 & 8 & 12 \\
\hline Crested Tern & * & & & & & 4 & 3 \\
\hline Black Noddy & * & & & & & 10 & \\
\hline Lesser Frigatebird & * & 1 & & 1 & & $1,000+$ & 20 \\
\hline Brown Booby & & & & & & 2 & \\
\hline Nicobar Pigeon & * & $5(3 i)$ & 4 & & 5 & & \\
\hline Stephan's Ground Dove & * & & 2 & 4 & & 2 & 1 \\
\hline Crested Cuckoo-dove & & $1^{*}$ & & & & & \\
\hline $\begin{array}{l}\text { Claret-breasted Fruit-dove } \\
\text { Superb Fruit-dove }\end{array}$ & * & $3+$ & 2 & 1 & & 4 & $\begin{array}{c}20+ \\
1\end{array}$ \\
\hline Red-knobbed Imperial Pigeon & * & $1+$ & 10 & & + & & 3 \\
\hline Island Imperial Pigeon & * & $10+$ & $100+$ & 10 & $+(\mathrm{b})$ & 20 & $20+$ \\
\hline Cardinal Lory & * & 6 & 50 & 20 & + & $50+$ & 10 \\
\hline Rainbow Lorikeet & * & 5 & $100+$ & 50 & & $10+$ & 8 \\
\hline Eclectus Parrot & * & 1 & 1 & & & 4 & 6 \\
\hline Finch's Pygmy-parrot & * & & & $2^{*}$ & & & \\
\hline Singing Parrot & & & & & & 2 & 4 \\
\hline Solomon Cockatoo & * & 12 & 5 & 4 & 1 & 6 & 4 \\
\hline Common Koel & & 1 & & 1 & & 2 & 2 \\
\hline Buff-headed Coucal & * & $5+$ & 3 & 3 & 3 & 4 & 3 \\
\hline Shining Bronze Cuckoo & & & & 1 & & & \\
\hline Glossy Swiftlet & * & 100 & 10 & 100 & & 5 & 4 \\
\hline Uniform Swiftlet & & & & 10 & & 12 & 15 \\
\hline Common Kingfisher & * & 2 & 3 & 2 & & 1 & \\
\hline Little/Mangrove Kingfisher & & & & & & & 2 \\
\hline Variable Kingfisher & & & 1 & & 1 & & \\
\hline Collared Kingfisher & * & & 1 & 2 & & 1 & 2 \\
\hline Beach Kingfisher & * & 2 & & 6 & 2 & 2 & 1 \\
\hline Dollarbird & * & & 1 & 1 & 2 & & \\
\hline Blyth's Hornbill & * & 2 & 2 & 1 & 2 & 4 & 5 \\
\hline White-throated Nightjar & $*(b)$ & & & & & 1 & \\
\hline Moustached Tree Swift & * & & & 1 & & 4 & 5 \\
\hline Pacific Swallow & * & 2 & 2 & & 6 & 2 & 4 \\
\hline White-bellied Cuckoo-shrike & * & & 2 & & 2 & 2 & 2 \\
\hline Yellow-eyed Cuckoo-shrike & * & & & & & 2 & 2 \\
\hline
\end{tabular}


TABLE 6 (continued)

\begin{tabular}{|c|c|c|c|c|c|c|c|}
\hline Common Name & 1999 & Qeuru & Erava & Tavara & Hokata & Lelei 2002 & Lelei 2003 \\
\hline Melanesian Cuckoo-shrike & & & & 1 & & & \\
\hline Common Cicadabird & & & & & & & 2 \\
\hline Yellow-vented Myzomela & & & 1 & 1 & & & 5 \\
\hline Common Golden Whistler & & & & 1 & & & \\
\hline Willie Wagtail & *(b) & 4 & 4 & $4(b)$ & 2(b) & 1 & 2 \\
\hline Rufous Fantail & & & & & & & 2 \\
\hline White-winged Fantail & & & & & 3 & & 1 \\
\hline White-capped Monarch & $*(\mathrm{~b})$ & & 4(b) & 4 & $10(\mathrm{~b})$ & & 8 \\
\hline Kolombangara Monarch & & 2 & 3 & 4 & 2 & & 1 \\
\hline Steel-blue Flycatcher & & 1 & & 4 & 1 & 2(b) & 2 \\
\hline Tetepare White-eye & * & 6 & 10 & 20 & 12 & $15(\mathrm{~b})$ & 20 \\
\hline Yellow-bellied Sunbird & * & 2 & 4(b) & 10 & $4(b)$ & 2 & 4 \\
\hline Yellow-faced Myna & * & 5 & 4 & 1 & 3 & 8 & 6 \\
\hline Singing Starling & & 20 & 4 & & 2 & 10 & \\
\hline Metallic Starling & * & & 4 & & $20+$ & & 15 \\
\hline Brown-winged Starling & & & & & & $3(\mathrm{~b})$ & 2 \\
\hline
\end{tabular}

* Unspecified location on Tetepare; (b), breeding record; (i), immature.

The 62 species of nonmigratory land birds recorded from Tetepare compares favorably with the 37 species recorded from the larger, yet more remote island of Rennell (Filardi et al. 1999) and the 76 resident species recorded from the very much larger Santa Isabel (Kratter et al. 2001), also in the Solomon Islands. Several rare species recorded on Tetepare are discussed later in this section. Sixteen additional resident avifauna species have been recorded from lowland forests in the Western Province by Mayr and Diamond (2001) augmented by C. Filardi (pers. comm.). In addition to the three regionally uncommon raptor and pigeon species, the most notable absentees from the Tetepare inventory are ground-dwelling crakes (Porzana spp.), rails (Gallirallus spp.), bush-hens (Amaurornis olivaceous), and ground-doves (Gallicolumba spp.). Further surveys, particularly in the dense vegetation around Lake Tavara and lowland swamps on the weather (southern) coast of Tetepare, may record some of these species, but it is possible that dense pig populations may have affected some ground-dwelling bird populations on Tetepare.

The Solomon Sea Eagle (Haliaeetus sanfordi), listed as a vulnerable bird species by BirdLife International (2000), was widespread throughout the island, and one individual was observed taking a Bare-backed Fruit-bat (Dobsonia inermis) that had been disturbed from its diurnal roost. Near-threatened species (BirdLife International 2000) recorded from Tetepare were Beach Thick-knee (Burbinus giganteus), Crested Cuckoo-dove (Reinwardtoena crassirostris), Nicobar Pigeon (Caloenus nicobarica), and Kolombangara Monarch (Monarcha browni). Several immature Nicobar Pigeons were observed, and it is likely that Tetepare's lowland forests are important feeding grounds for this declining species that nests colonially on nearby Ngirasa Island. Tetepare is also an important feeding ground for Island Imperial Pigeons (Ducula pistrinaria) that fly to Tetepare in their thousands from adjacent nesting atolls. The Kolombangara Monarchs on Tetepare had considerably larger and squarer white cheek patches than those illustrated in Doughty et al. (1999). The Crested Cuckoodove record was a solitary individual observed at close quarters flying near the coast. Our guides had seen this distinctive species on Tetepare only once before.

White-throated Nightjars (Eurostopodus mystacalis) were recorded breeding at Keife in October 1999, and several other individuals were recorded in near-coastal habitats on Tetepare. The scarcity of cats and dogs may render Tetepare an important refuge 
for these beach-nesting birds, believed to possibly represent a threatened species, endemic to the Solomons (G. Dutson, pers. comm.). Two Little Pied Cormorants (Phalacrocorax melanoleucos), which also inhabit the larger Lake Rano on adjacent Rendova, were recorded on Lake Tavara on Tetepare for the first time. Despite only colonizing the major islands of the New Georgia group of islands since the 1940s (Mayr and Diamond 2001), Papuan Hornbills (Rhyticeros plicatus) were widespread and relatively abundant on Tetepare (Dutson 2001; this study).

The Tetepare White-eye (Zosterops rendovae tetiparius) was easily distinguished from all other white-eyes in Doughty et al. (1999) by the combination of absence of a white eye ring; black bill; black mask from bill to eye; distinct green bib; gray-white belly; yellow on the bend of the wing, underside of thighs, and tail; and yellow legs. Live specimens differed from the illustration in Mayr and Diamond (2001) by the prominent yellow patch on the bend of the wing that was clearly visible on all of the hundreds of individuals observed. This yellow patch on the wing and the absence of a yellow belly clearly distinguish it from its sister subspecies on Rendova, less than $3 \mathrm{~km}$ away. Zosterops $r$. tetiparius was abundant in coastal, riverside, and regenerating forest throughout Tetepare and was also recorded from inland forest canopy.

Extensive rocky tidal flats on both the western and southeastern extremities of Tetepare may prove to be valuable staging grounds for migratory waders. Whimbrels (Numenius phaeopus), Mongolian Plovers (Charadrius mongolus), Pacific Golden Plovers (Pluvialis fulva), Red-necked Stints (Calidris ruficollis), Gray-tailed Tattlers (Tringa brevipes), and Common Sandpipers (Tringa bypoleucos) were recorded in these habitats during these brief surveys.

The dense populations of frogs on Tetepare may be indicative of the unpolluted rivers and the absence of Cane Toads (Bufo marinus). Cane toads are believed to have been introduced to the western Solomons during World War II and are now abundant at Munda, Gizo, and northern and western Rendova and have recently been introduced to the Rendova village of Lokuru, adjacent to Tetepare. If introduced to Tetepare, these poisonous toads have the potential to seriously affect native fauna populations. We recorded one cat from the Lelei region, but pig hunters report that feral cats were occasionally found throughout the island. The control of these pests should assist in the conservation of many vertebrates, particularly ground-nesting bird species.

The fauna species and communities of note identified by these preliminary vertebrate surveys of Tetepare, combined with the continued extensive logging of adjacent islands, suggest that Tetepare may be a valuable refuge for many species of conservation concern. More detailed bat surveys are a priority, especially considering the records of a potentially different Hipposideros taxon on Tetepare (Gee 2003) and Melonycteris fardoulisi that was only described in 1993 (Flannery 1995). A recently discovered Pteralopex flying fox, the New Georgia Monkey-faced Bat, is also apparently restricted to lowland primary rain forest in the vicinity of Tetepare (Fisher 1992).

Our surveys confirm that Tetepare Island remains a largely untouched wilderness island, with considerable conservation value on regional, national, and international scales. Of particular note are the large stands of lowland and coastal forest, habitats that have been extensively exploited for plantations, gardens, and logging elsewhere in the Solomon Islands. With the assistance of international scientists and ecotourists, the conservation and resource management plan currently being implemented by the Tetepare Descendants' Association should help to maintain the increasingly valuable biological communities, traditional knowledge, and bush resources of Tetepare Island.

\section{ACKNOWLEDGMENTS}

The Tetepare Descendants' Association (TDA) and its precursor, the Friends of Tetepare, granted permission to visit, encouraged research on Tetepare, and provided logistical support with canoe and field station accommodation during the later surveys. 
TDA is supported financially by NZAid and European Union Microprojects. We thank guides and field assistants Mary Bea, Twomey Ben, Keto Hebale, John Tume, Edwin Lau, Suhero, Matthias Daly, Vanja Rohwer, Hobete, and William Kodo for their efforts in the field and Isaac Molia for arranging our initial visit to Tetepare. David Gee conducted a pilot bat survey on Tetepare in August 2003, Steve Richards assisted with the identification of amphibians and reptiles, and we thank Chris Filardi, Catherine Smith, Guy Dutson, and Jared Diamond for sharing their bird observations on Tetepare and commenting on the manuscript.

\section{Literature Cited}

Austin, C. C., and K. W. Jessing. 1994. Green-blood pigmentation in lizards. Comp. Biochem. Physiol. 109:619-626.

Beehler, B. M., T. K. Pratt, and D. A. Zimmerman. 1986. Birds of New Guinea. Princeton University Press, Princeton, New Jersey.

BirdLife International. 2000. Threatened birds of the world. Lynx Edicions and BirdLife International, Barcelona and Cambridge, United Kingdom.

Dahl, A. 1980. Regional ecosystem survey of the South Pacific area. SPC/IUCN Technical Paper 179. South Pacific Commission, Noumea.

Doughty, C., N. Day, and A. Plant. 1999. Birds of the Solomons, Vanuatu and New Caledonia. Christopher Helm Publishers, London.

Dutson, G. 2001. New distributional ranges for Melanesian birds. Emu 101:237-248.

Filardi, C. E., C. E. Smith, A. W. Kratter, D. W. Steadman, and H. P. Webb. 1999. New behavioral, ecological, and biogeographic data on the avifauna of Rennell, Solomon Islands. Pac. Sci. 53:319-340.

Fisher, D. 1992. An ecological study of a new species of monkey-faced bat from the islands of New Georgia and Vangunu, the Solomon Islands. Unpubl. report (available from Australian Museum, Sydney).

Flannery, T. 1995. Mammals of the South West Pacific and Moluccan Islands. Australian Museum/Reed Books, Chatswood, New South Wales, Australia.

Gee, D. 2003. Bat survey of Tetepare Island. Tetepare Descendants' Association Technical Report 2. World Wildlife Fund, Solomon Islands.

Hansell, J. R. F., and J. R. D. Wall. 1976. Land resources of the Solomon Islands. Land Resource Study 18. Land Resources Division, Ministry of Overseas Development, Surrey, England.

Hviding, E. 1995. Of reef and rainforest: A dictionary of environment and resources in Marovo Lagoon. University of Bergen, Bergen, Norway.

Kratter, A. W., D. W. Steadman, C. E. Smith, C. E. Filardi, and H. P. Webb. 2001. Avifauna of a lowland forest site on Isabel, Solomon Islands. Auk 118:472483.

Lees, A. 1990. A protected forests system for the Solomon Islands. Maruia Society, Nelson, New Zealand.

Mayr, E., and J. Diamond. 2001. The birds of northern Melanesia: Speciation, ecology, and biogeography. Oxford University Press, Oxford.

McCoy, M. 2000. Reptiles of the Solomon Islands. CD, Zoographics, Kuranda, Australia.

Spotila, J. R., R. D. Reina, A. C. Steyermark, P. T. Plotkin, and F. V. Paladino. 2000. Pacific leatherback turtles face extinction. Nature (Lond.) 405:529-530.

SPREP (South Pacific Regional Environment Programme). 1985. Country review, Solomon Islands. 3rd SPREP conference. South Pacific Commission, Apia, Western Samoa. 
